EPJ Web of Conferences 82, 01050 (2015)

DOI: $10.1051 /$ epjconf/20158201050

(C) Owned by the authors, published by EDP Sciences, 2015

\title{
Characteristics of standing disperse medium on the filter substrate
}

\author{
Michail Vasilevsky, Aleksandr Razva a and Ulukbek Sattarov \\ National research Tomsk polytechnic university, 634050 Tomsk, Russia
}

\begin{abstract}
In the process of gas filtration through a porous wall deposited dust is agglomerated state. Sealing layer in the accumulation of particles associated with the reformation of aggregates. Kozeny equation Karman reflects the relationship between flow rates, porosity layer, a pressure drop, a specific surface area of the particles in the layer thickness. The specific surface area of the particles in the layer is an unknown quantity, so it is proposed to determine the ratio of Kozeny Karmana obtained from experiments on all other values. Filtering process in the experimental setup was performed on preformed compressible layers of particles of different size. Thus the filtration rate is fixed, the change in porosity, the pressure drop, the thickness of the layer. It is believed that the specific surface of the particles remains constant when implementation filtration processes similar manufacturing conditions.
\end{abstract}

\section{Introduction}

In systems scrubbing gas filtration through a bed of particles is the most effective means of dedusting gases. As Deduster apply flexible partitioning of tissue metal grids. Dedusting process gas is formed in the layer of the filtered particles [1]. Mechanical characteristics of the formed layer of particles is determined by the methods of regeneration of the filter walls, on which depends the efficiency, stability, dedusting, reliable operation of the filter.

\section{Theoretical bases}

In [2] provides an analysis of methods for determining the resistivity of the sediment. The total resistance $R_{\Sigma}=R_{\mathrm{sl}}+R_{\mathrm{f}},(1 / \mathrm{m})$. For incompressible layer and filtering partition $P_{\Sigma}=\mu R_{\Sigma} W,(\mathrm{~Pa})$, where $\mu$ - the dynamic viscosity, (Pa s). The magnitude $R_{\mathrm{sl}}=r_{0} H_{\mathrm{k}}$ where $r_{0}$ value of resistivity layer $\left(1 / \mathrm{m}^{2}\right)$.

In [2] does not address the issue of the stresses arising in the formed on the porous layer of the septum, which depend on the pressure drop. Tension in the particulate body increases towards the septum, thus decreasing porosity layer and the resistivity increases. In [2], the resistivity of the layer height is taken averaged constant, but depends on the pressure. Defined mechanical characteristics

\footnotetext{
a Corresponding author: razva@mail.ru
}

This is an Open Access article distributed under the terms of the Creative Commons Attribution License 4.0, which permits unrestricted use, distribution, and reproduction in any medium, provided the original work is properly cited. 


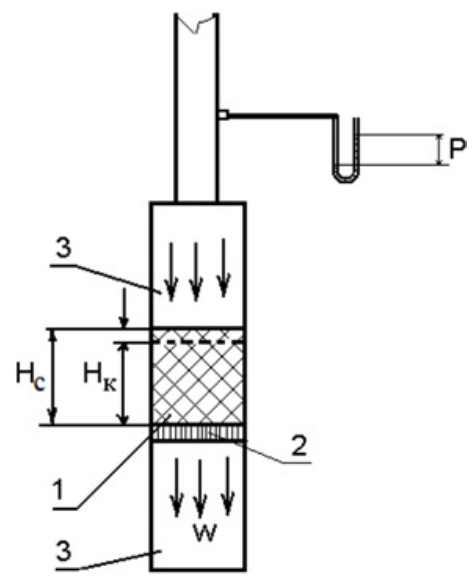

Figure 1. Device for determining the parameters of the particulate material (1-working section, 2-filtr, 3-auxiliary section).

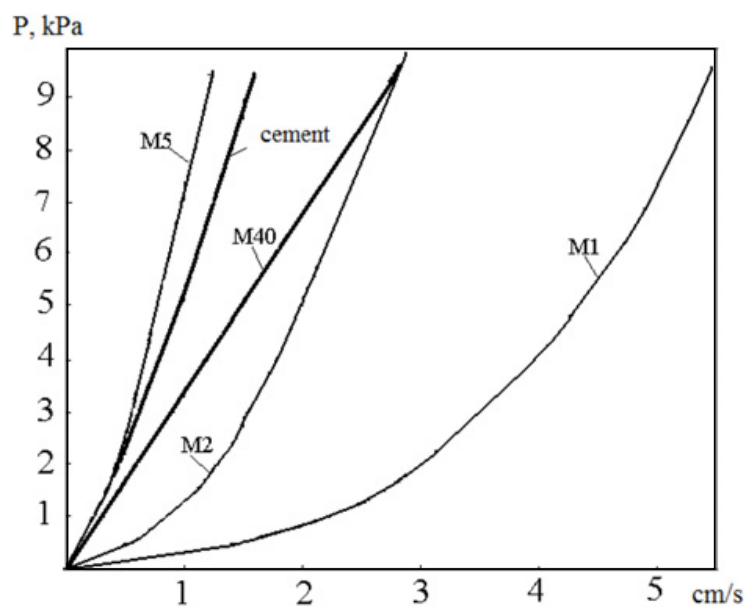

Figure 2. The air pressure to the working cylinder of height $4.8 \mathrm{~cm}$ from the filtration rate.

dispersed bodies formed into cylinders backfilling fine powder consisting of agglomerates of particles (Fig. 1) [3].

\section{Experimental setup description}

It was found that the bulk density in the cylinder is dependent on the method of preparation of a filling material, the relative humidity, the material of the cylinder, the height of the cylinder. Figures 2 and 3 show the experimental dependence of pressure change of the filtration rate and change in porosity of pressure for different powders of metal cylinder height $48 \mathrm{~mm}$ with one and the same filter fabric, which after each run was freed from the particle bed. The material after sieving through a sieve with a mesh of $1 \mathrm{~mm}^{2}$ consists of agglomerated particles and after filling into the working cylinder has a bulk density which is caused autohesive interactions between the particles. 


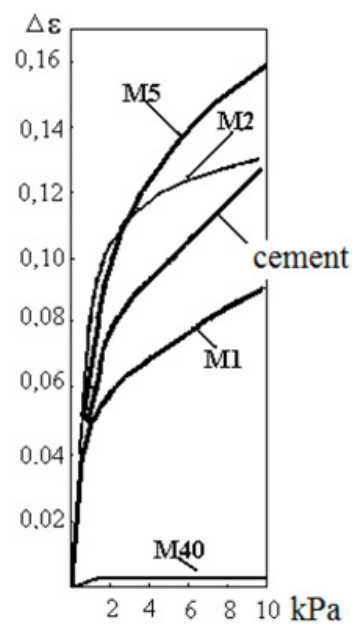

Figure 3. Changing the average porosity of the pressure.

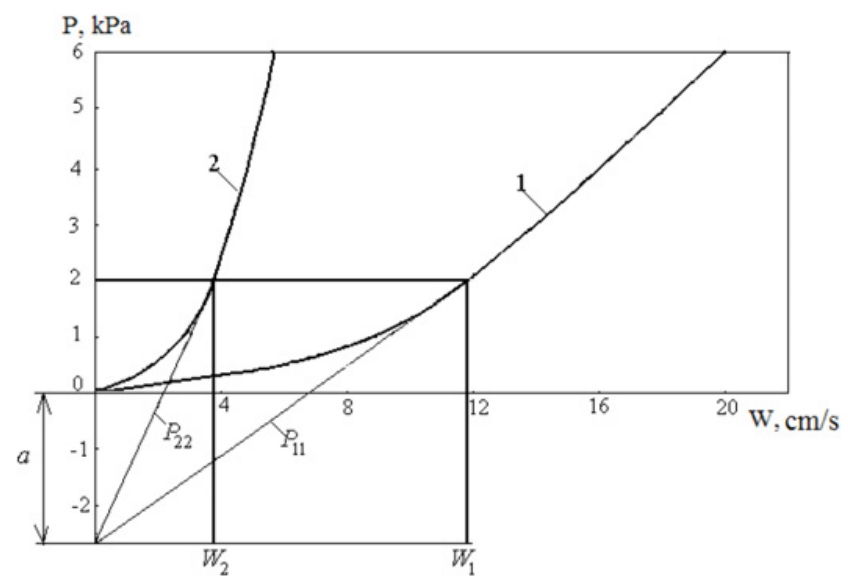

Figure 4. Dependence of the inlet pressure in the cylinders of the air velocity in the cylinders. $\mathrm{P}_{11}, \mathrm{P}_{22}$ line pressures approximating the real changes in the range of 1.5 to $2.5 \mathrm{kPa}$, with initial coordinate point "a".

\section{Experimental technique and discussion and conclusions}

Assessment redistribution resistances between the filter layer and consider the example air filtration through a layer of powder M2 in the cylinders 10 and height $40 \mathrm{~mm}$ [3]. Figure 4 shows the curves 1 and 2, depending on the rate of pressure cylinders 10 and height $40 \mathrm{~mm}$.

Curves 1 and 2 show that the compressible layer. Draw a line $P=S=$ const so that the tangent to the lines 1 and 2 at the points of intersection intersect the ordinate axis at $\mathrm{a}_{1} \approx \mathrm{a}_{2} \approx \mathrm{a}=2.68 \mathrm{kPa}$ (Fig. 4). Draw curve fitting in the pressure range $P=1.5-2.5 \mathrm{kPa}$ follows:

$$
\begin{gathered}
\left.\frac{d P_{2}}{d W}\right|_{p=s}=\mu R_{\Sigma 2}=\frac{S}{W_{2}} \\
\left.\frac{d P_{1}}{d W}\right|_{p=s}=\mu R_{\Sigma 1}=\frac{S}{W_{1}} . \\
01050-\text { p.3 }
\end{gathered}
$$




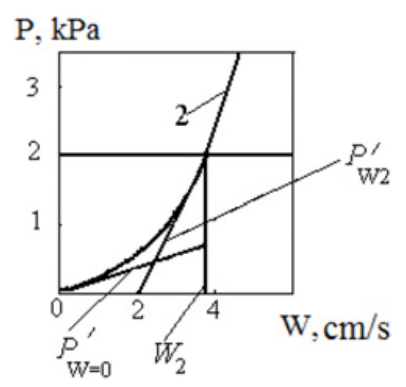

Figure 5. Detection circuit derivatives.

In the pressure range $P=1.5-2.5 \mathrm{kPa}$, this line corresponds to the value $P=S \approx 2 \mathrm{kPa}$. Curves of pressure changes on the rate for incompressible layers represent linear dependencies $P_{11}=\mu R_{\Sigma 1} W$, $P_{22}=\mu R_{\Sigma 2} W, P_{11}, P_{22}$ air pressure in front of a height of $N_{K 1}$ and $N_{K 2}$ with initial coordinate linear approximations of the point a, $R_{\Sigma 1}, R_{\Sigma 2}$ - resistance layer height $H_{k 1}$ and $N_{K 2}$, respectively. These relations imply

$$
\mathrm{R}_{\Sigma 1}=\frac{\mathrm{P}_{11}}{\mu \mathrm{W}}, \mathrm{R}_{\Sigma 2}=\frac{P_{22}}{\mu W}, r_{0}=\frac{R_{\Sigma 2}-R_{\Sigma 1}}{H_{k 2}-H_{k 1}}, \mathrm{R}_{\theta}=\frac{R_{\Sigma 1} H_{k 2}-R_{\Sigma 2} H_{k 1}}{H_{k 2}-H_{k 1}} .
$$

We have the following data to calculate: $H_{k 1}=10 \cdot(1-\Delta \varepsilon) \cdot 10^{-3}=10 \cdot(1-0,1) \cdot 10^{-3}=9 \cdot 10^{-3}$, $\mathrm{m}$; similarly $H_{\mathrm{k} 2}=40 \cdot(1-\Delta \varepsilon) \cdot 10^{-3}=40 \cdot(1-0,1) \cdot 10^{-3}=36 \cdot 10^{-3}, \mathrm{~m} ; \mu=1,8 \cdot 10^{-5}, \mathrm{~Pa} \cdot \mathrm{s}$. According to Fig. 4 , we have the following parameters: $W_{1}=11.8 \cdot 10^{-2}, \mathrm{~m} / \mathrm{s} ; W_{2}=3.8 \cdot 10^{-2}, \mathrm{~m} / \mathrm{s}$; $P_{22 \mathrm{~s}}=(a+2) \cdot 10^{3}=P_{11 \mathrm{~s}}=4.68 \cdot 10^{3}, \mathrm{~Pa}$. Substituting these data in the quantities appearing in the formula (1), we obtain $R_{\Sigma 1}=2.2 \cdot 10^{9}, 1 / \mathrm{m} ; R_{\Sigma 2}=6.8 \cdot 10^{9}, 1 / \mathrm{m} ; R_{f}=0.67 \cdot 10^{9}, 1 / \mathrm{m} ; r_{0}=1.7$. $10^{11}, 1 / \mathrm{m}^{2}$. Note that pressure in the range $P=1.5-2.5$, kPa determined by the relations $P_{2}=P_{22}-$ $2.68, \mathrm{kPa} ; P_{1}=P_{11}-2.68, \mathrm{kPa}$.

Suppose that after the filtering process dispersed body consists of compacted and uncompacted parts. To evaluate the distribution of seals and unconsolidated portions along the length of the body of dispersed use of curve 2 in Fig. 4. Denote $\left.P^{\prime}\right|_{\mathrm{W}=0}=\left.(d P / d W)\right|_{\mathrm{W}=0},\left.P^{\prime}\right|_{\mathrm{W} 2}=\left.(d P / d W)\right|_{\mathrm{W} 2}-$ derivative at $W=W_{2}$ (Fig. 5).

We write for filtering particulate compacted and non-compacted body in the form of $\left.P^{\prime}\right|_{\mathrm{W}=0}=$ $H_{\mathrm{k}} \mu r_{0 \mathrm{H}},\left.P^{\prime}\right|_{\mathrm{W} 2}=H_{\mathrm{k}} \mu r_{0 \mathrm{y}}$. The total pressure is made up of the pressure loss of the particulate unconsolidated portion of the body and the pressure loss at the portion of the seal body. $P=$ $W_{2}\left(h_{H} \mu r_{0 H}+\left(H_{k}-h_{H}\right) \mu r_{0 y}\right)$ or

$$
P=W_{2}\left[\left.P^{\prime}\right|_{W=0} \frac{h_{H}}{H_{k}}+\left.P^{\prime}\right|_{W 2} \frac{H_{k}-h_{H}}{H_{k}}\right]
$$

where $h_{\mathrm{H}}-$ the height of the unconsolidated particulate body, whence:

$$
\left.\left.h_{H} / H_{k}=\left[\left.P^{\prime}\right|_{W 2}-\left(P / W_{2}\right)\right] /\right]\left.P^{\prime}\right|_{W 2}-\left.P^{\prime}\right|_{W=0}\right] .
$$

For example in Fig. 5 should $\left.P^{\prime}\right|_{W 2}=2 /(3.8-2.2)=1.25 ;\left.\quad P^{\prime}\right|_{W=0}=0.75 / 4=0.19 ; \quad P / W_{2}=$ $2 / 3.8=0.52$. Substituting these values in the formula (5), we obtain $h_{\mathrm{H}} / \mathrm{H}_{k}=0,69$. Consequently, the height of the compacted particulate body is less than a third of the height of the cylinder. These data allow us to evaluate the stresses in the particle layer. 
Thermophysical Basis of Energy Technologies

\section{References}

[1] M.G. Mazus, A.D. Malgin, M.L. Morgulis. Filters to trap industrial dusts (Engineering, Moscow, 1985)

[2] V. Zhuzhikov. Filtration: Theory and practice of suspensions separation (Chimia, Moskva, 1980)

[3] M.V. Wasilevsky, V.I. Romandin, A.S. Razva, E.G. Zykov, V.A. Polyushko. The method of determining the characteristics of the layer of particulate material on a membrane filter (Proceedings of the Eighteenth All-Russian Scientific and Technical Conference "Energy: efficiency, reliability, and security", Tomsk, 2012) 